\title{
Proanthocyanidins-Rich Salacia Extract Enhances Glucose Uptake and Glycogen Content in Human Skeletal Muscle Cells \\ Khadija Ghanam $^{1}$, Sandhya Nair ${ }^{1}$, Jayant Deshpande ${ }^{1,2}$, Vijaya Juturu ${ }^{2}$ \\ ${ }^{1}$ Omniactives Health Technologies Ltd, Charlottetown, PEI, Canada. \\ 2 Omniactives Health Technologies Inc, Morristown, NJ, USA.

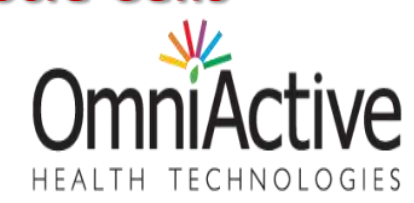

\section{INTRODUCTION}

- $80 \%$ of insulin-sensitive glucose metabolism occurs in skeletal muscle cells. An understanding of the mechanism and regulation of muscle glucose utilization is necessary to determine the nature of the defects present in metabolic diseases like obesity and insulin-resistant diabetes.

- Glucose transport is the initial step, and often considered rate determining, in glucose utilization in the muscle.

- Uptake of glucose and glycogen synthesis in skeletal muscle is of interest for type 2 diabetes treatment.

- Salacia extracts have been used in traditional Ayurvedic medicine for generations and has been extensively consumed as a food supplement for the prevention of diabetes.

\section{OBJECTIVE}

This study aimed to determine the effects of various concentrations of proanthocyanidins-rich Salacia extract $(1,10$ and $100 \mu \mathrm{g} / \mathrm{ml})$ on insulin stimulated glucose uptake, glycogen accumulation and ATP synthesis in primary human skeletal muscle cells.

\section{METHODOLOGY}

- Human skeletal muscle cells (SkGM CC-2561, Lonza Walkersville, Inc.USA ) were subcultured according to Lonza protocol, using BulletKit growth medium (CC-3160 , Lonza, USA) and Reagent Pack ${ }^{\text {TM }}$ Subculture Reagents (CC-5034, Lonza, USA).

- Glucose uptake in human skeletal muscle cells was determined by a bioluminescent assay using a glucose uptake assay kit (CS1876A01, Promega, USA), based on the detection of 2-deoxyglucose-6phosphate. The luminescence signal was proportional to the concentration of 2deoxyglucose-6-phosphate.

- Glycogen synthesis was determined in cell culture lysates using a colorimetric assay kit (K-646-100, BioVision, Inc, USA). Absorbance was measured at $570 \mathrm{~nm}$.

- ATP levels in muscle cells were assessed using an ATP colorimetric assay kit (MAK190, Sigma-Aldrich, USA).

- In all assays, the effect of Proanthocyanidins-rich Salacia extract was compared to the effect of two positive controls: caffeine $(100 \mu \mathrm{g} / \mathrm{ml})$ and leucine $(100 \mu \mathrm{g} / \mathrm{ml})$.

\section{RESULTS}

A- Salacia extract (SE) significantly increased glucose uptake in the presence of insulin in muscle cells.

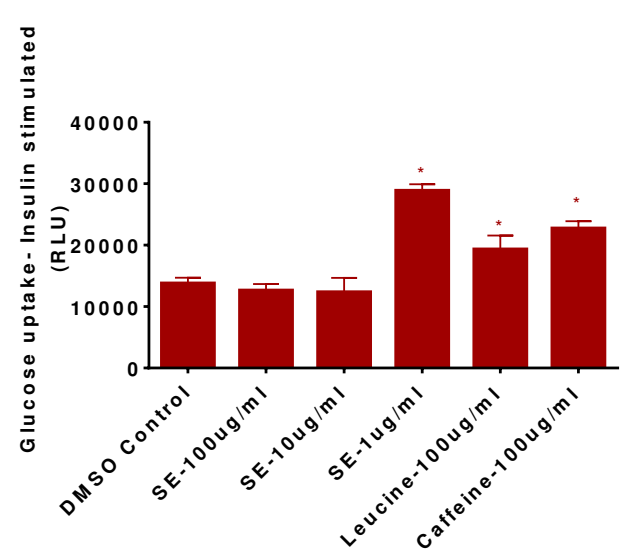

B- Salacia extract (SE) significantly enhanced glycogen content in the presence of insulin in muscle cells.

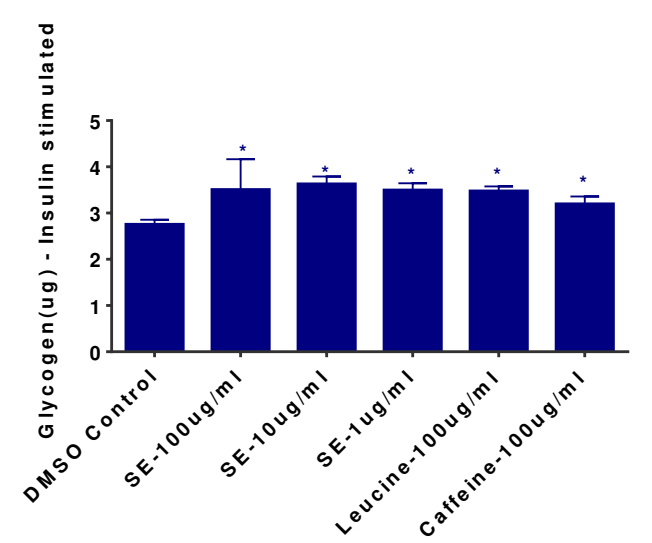

C- Salacia extract (SE) significantly enhanced ATP synthesis in muscle cells.

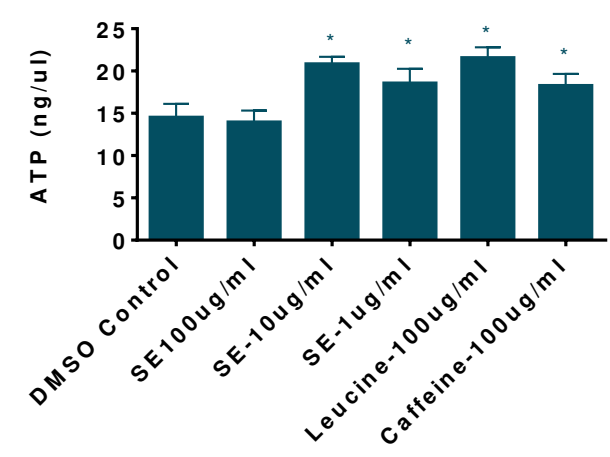

\section{CONCLUSION}

- Salacia extract significantly enhanced glucose uptake and glycogen content in muscle cells compared to vehicle control.

- Salacia extract also enhanced ATP synthesis in muscle cells.

- Salacia extract is a promising nutraceutical ingredient that may improve insulin resistance by enhancing glucose utilization in skeletal muscles. 